\title{
Building Telemedicine Capacity for Trainees During the Novel Coronavirus Outbreak: a Case Study and Lessons Learned
}

\author{
Katharine Lawrence, MD MPH ${ }^{7}$ (1), Kathleen Hanley, MD², Jennifer Adams, MD², \\ Daniel J Sartori, MD², Richard Greene, MD MHPE' ${ }^{2}$, and Sondra Zabar, $\mathrm{MD}^{2}$
}

'Department of Population Health, NYU Grossman School of Medicine, New York, NY , USA; ${ }^{2}$ Department of General Internal Medicine and Clinical Innovation, NYU Grossman School of Medicine , New York , NY , USA.

INTRODUCTION: Hospital and ambulatory care systems are rapidly building their virtual care capacity in response to the novel coronavirus (COVID-19) pandemic. The use of resident trainees in telemedicine is one area of potential development and expansion. To date, however, training opportunities in this field have been limited, and residents may not be adequately prepared to provide high-quality telemedicine care.

AIM: This study evaluates the impact of an adapted telemedicine Objective Structured Clinical Examination (OSCE) on telemedicine-specific training competencies of residents.

SETTING: Primary Care Internal Medicine residents at a large urban academic hospital.

PROGRAM DESCRIPTION: In March 2020, the New York University Grossman School of Medicine Primary Care program adapted its annual comprehensive OSCE to a telemedicine-based platform, to comply with distance learning and social distancing policies during the COVID-19 pandemic. A previously deployed in-person OSCE on the subject of a medical error was adapted to a telemedicine environment and deployed to 23 primary care residents. Both case-specific and core learning competencies were assessed, and additional observations were conducted on the impact of the telemedicine context on the encounter.

PROGRAM EVALUATION: Three areas of telemedicine competency need were identified in the OSCE case: technical proficiency; virtual information gathering, including history, collateral information collection, and physical exam; and interpersonal communication skills, both verbal and nonverbal. Residents expressed enthusiasm for telemedicine training, but had concerns about their preparedness for telemedicine practice and the need for further competency and curricular development.

DISCUSSION: Programs interested in building capacity among residents to perform telemedicine, particularly during the COVID-19 pandemic, can make significant impact in their trainees' comfort and preparedness by addressing key issues in technical proficiency, history

Electronic supplementary material The online version of this article (https://doi.org/10.1007/s11606-020-05979-9) contains supplementary material which is available to authorized users.

Received March 27, 2020

Revised May 15, 2020

Accepted June 11, 2020

Published online July 8, 2020 and exam skills, and communication. Further research and curricular development in digital professionalism and digital empathy for trainees may also be beneficial.

KEY WORDS: telemedicine; residency training; Objective Structured Clinical Examination (OSCE); standardized patient; clinical skills assessment; coronavirus; COVID-19.

J Gen Intern Med 35(9):2675-9

DOI: $10.1007 / \mathrm{s} 11606-020-05979-9$

(c) Society of General Internal Medicine 2020

\section{BACKGROUND}

The past several months have seen unprecedented changes in the field of virtual healthcare ${ }^{1}$. As a result of the novel coronavirus (COVID-19) pandemic and the social distancing policies that have been put into place in an attempt to reduce viral spread, both hospital and ambulatory care systems have made enormous efforts to expand and fortify their virtual care capacity. These efforts parallel policy changes at both the state and national level, where there is growing recognition of the urgent need to support remote care delivery. On March 6, 2020, the Centers for Medicare and Medicaid Services (CMS) broadened access to "telehealth," including a waiver to allow for reimbursement of telemedicine services across the country, regardless of patient or provider location (i.e., across states) or whether the provider is physically located at a healthcare facility ${ }^{1}$. Subsequent policies from CMS, as well as major insurers and regulatory agencies, have continued to facilitate this rapid adoption and expansion.

Across academic institutions, where much of the physician workforce is comprised of trainees such as residents, discussions are ongoing as to how best to protect learners while minimizing disruptions in clinical training and care delivery. One area of exploration is that of telemedicine, where the

\footnotetext{
'The terms "virtual healthcare," "telehealth," and "telemedicine" are often used interchangeably. In practice, telemedicine generally refers to a subset of telehealth or virtual health services and is defined by CMS as the "two-way, real time interactive communication between the patient and the physician or practitioner at [a] distant site." For the purposes of this article, and to reflect the vocabulary used in the majority of research and training on the topic, we refer to telemedicine throughout.
} 
safety of physician-trainees can be assured even as quality medical care, patient triage, and education take place. Recently, the ACGME accelerated the Common Program Requirements for supervision of telemedicine visits carried out by trainees, to permit residents and fellows to conduct telemedicine to care for patients affected by the pandemic ${ }^{2}$. While telemedicine has been increasingly recognized by major medical organizations (including the AMA and ACGME) as a needed competency among physician-trainees, training opportunities in this field have thus far been limited ${ }^{3-5}$. As a result, residents may not be adequately prepared to provide highquality care via telemedicine, and may feel it is beyond their scope to do so.

In March 2020, the New York University Grossman School of Medicine Primary Care program had a unique opportunity to witness residents performing telemedicine visits in a structured, supervised environment. In an effort to implement social distancing policies and mitigate the spread of the novel coronavirus within the community, the program moved its annual Primary Care Comprehensive Objective Structured Clinical Examination (OSCE) - a day-long multi-station simulation event for residents that includes recorded clinical encounters with trained Standardized Patients (SPs) and comprehensive learner feedback from faculty - to a fully telemedicine-based platform. Five of the originally planned 11 OSCE cases were adapted to a "virtual visit" telemedicine format, with learners and SPs interacting remotely over a webbased platform installed in the simulation space. One OSCE case - an encounter regarding a misdiagnosis in the "OpenNotes" electronic health record-had been recently conducted as an in-person encounter and was re-deployed as a telemedicine visit. The OpenNotes in-person and virtual case study offers timely learning points for our own teaching program, as well as insights for other institutions who are interested in quickly building and scaling their telemedicine capacity among residents and other trainees.

\section{METHODS}

The OpenNotes OSCE case involved a middle-aged woman who accesses her complete electronic health record (EHR) through the patient-facing OpenNotes function, and sees a diagnosis of "major depressive disorder" in her medical history that she feels is inaccurate. She makes several inquiries about her concern through the patient portal messaging system with no response and ultimately presents to clinic to discuss her concerns. Trainee case learning objectives were to address the patient's concerns regarding perceived errors in the EHR, to clarify relevant medical history, to recognize opportunities and limitations of patient-facing EHR tools, and to set expectations around appropriate use of these tools. Competencies assessed included acknowledging a potential medical error, recognizing and addressing patient emotions, and establishing trust.
Trained SPs were provided with a standardized evaluation tool that evaluated learners across a number of domains and rated their performance using a behavioral anchored scale (not done, partially done, and done) $)^{7}$. Core competency domains included communication (information gathering, rapport building, and patient education), patient satisfaction, and patient activation. Case-specific items were also evaluated and were developed based on best-practice guidelines for OpenNotes and patient portal usage, as well as the clinical specifics of the case (see Appendix 1. Faculty observers gave verbal feedback immediately after the case and completed a one-item entrustment scale. Written SP and faculty evaluations were provided to learners after completion of the Comprehensive OSCE experience (see Appendix 2).

The OSCE was conducted in the NYSIM Center using simulated clinical rooms with audio and video recording capability and one-way mirrors for faculty observation. An initial in-person encounter was completed by a subset of primary care residents based on scheduling, followed by an adapted telemedicine-based encounter with a second group of residents. In the telemedicine-based encounter, learner and SP were placed in separate clinical rooms and connected via video screens, with faculty observers monitoring through a one-way mirror. Both the in-person and telemedicine-based case were $10 \mathrm{~min}$, with $5 \mathrm{~min}$ of feedback from faculty observers, and standardized assessments completed by both SPs and faculty.

\section{RESULTS}

Thirty-two medicine residents completed the case: 9 in the inperson cohort and 23 in the subsequent telemedicine cohort. The following observations were made by learners, faculty, and SPs regarding the impact of the telemedicine environment on the patient encounter. They address key issues in telemedicine care, including technical proficiency, virtual information gathering (including history-taking and physical exam skills), and interpersonal communication competencies.

\section{Technical Competencies}

A key component of a successful telemedicine visit lies in the provider's technical proficiency. Most major medical associations recommend at least basic training in technical elements such as screen location, room lighting, audio adjustments, and computer system optimization ${ }^{8}$. It is also advisable to introduce new patients to the virtual clinic space and set expectations around the scope and limitations of a telemedicine visit. In our cohort, the majority of learners either did not acknowledge the novel virtual encounter or did not assess for functionality of its various technical aspects. Only two of the 23 learners explicitly addressed the use of the telemedicine space ("Welcome to the virtual encounter") or asked the SP if they had familiarity with telemedicine ("Have you done a televisit before?"). One learner, in the context of addressing disruptive technology in medicine in general, discussed the impact of 
telemedicine on patient care ("These are new technologies, and we are all learning together"). Four learners asked if the audio and visual features were optimized, and none of the learners asked the SP to adjust their screen when unable to fully see the patient. Of note, no technical issues with the telecommunications tools were observed during the encounters, and thus, learners were not observed addressing issues such as poor connection or audio/visual delays. One learner required an adaptive device (personal headphones) to facilitate their encounter, which did not impact the learning environment but required changes in the faculty observer's setup.

\section{History-Taking, Collateral Information, and Physical Exam}

A unique challenge of telemedicine is the adaptation of traditional components of the medical history and physical exam into the virtual space. Providers may be unable or unaccustomed to acquiring basic information from remote patients, such as home vital signs or medication review. And, while many physical exam maneuvers can be self-executed by patients and communicated to physicians in real-time, the diagnostic accuracy of these maneuvers is not yet known. In our cohort, learners were universally able to execute basic historytaking and mental health screening with their SP. All learners asked clarifying information about the patient's history of depression (with nineteen completing PHQ2 or PHQ9s), and many were able to obtain a complete medical, surgical, and social history. However, no learner actively utilized the patient's "home" environment to obtain collateral information. In this case, the SP had been provided with a printed simulated after-visit summary (AVS), to which she referred throughout the case and frequently showed the learners on-screen. No learner asked to see the file in more detail, had the patient read from the file, or asked about additional files or documents in the home. In comparison, all learners in the in-person OSCE asked to review the printed AVS form, and many asked additional questions regarding other materials they saw in the document. Of note, while a physical exam was not a requirement for this case, no learner offered to perform one or discuss how one might be performed in a virtual context.

\section{Communication Skills}

Communication is another area that presents unique challenges in the telemedicine environment. While verbal communication can be facilitated by seamless connectivity and high-quality visual displays, technical challenges can result in significant barriers to communication. Research also suggests that the virtual environment itself poses challenges to classical models of patient-provider communication ${ }^{9}$. Additionally, many traditionally employed nonverbal cues (such as allowing for silence, open posturing, and empathetic touch) may be difficult to deploy and/or interpret, both by patients and providers, in a virtual context ${ }^{10}$. In this OSCE, differences in both verbal and nonverbal communication strategies were observed in the telemedicine cohort as compared with the in-person cohort. During the encounter, several instances of the learners and SP speaking over each other and/or interrupting without apparent intention to do so were noted; while this was not significantly different from the in-person cohort, the responses to the interruptions were less fluent and appeared more disruptive to the encounter (in one instance, the SP commented directly "sorry I'll shut up now"). During feedback with the faculty, the SP endorsed feeling that the telemedicine learners spent overall more time talking than their in-person colleagues and that there were fewer opportunities to answer questions or offer more information during the case ("I felt like I couldn't get a word in sometime"). During observation, only one learner was noted to employ "pausing for silence" for greater than $5 \mathrm{~s}$, compared with more regular usage in the in-person encounter. In observing nonverbal communication, 21 learners sat forward in their seats, mirroring "leaning in" posturing with their patient. During feedback, learners acknowledged the importance of eye contact in the visit, but often expressed uncertainty as to the best place to "look" at their patients. Many felt the most natural place was to look at the patient's face on the screen. One learner stated they "looked" at the camera lens, but did not know if that was correct, and expressed some distraction about seeing their own image in the bottom corner of the screen.

During the group debriefing of the OSCE, residents expressed general enthusiasm for the idea of telemedicine and identified need within their own training for further competency and curricular development. They also expressed significant concern about their overall preparedness to conduct telemedicine visits and their ability to provide high-quality care to their patients.

\section{DISCUSSION}

The adaptation of our Primary Care Comprehensive OSCE to a telemedicine platform offered a unique opportunity to observe resident performing telemedicine visits across a variety of structured cases. Additionally, the implementation of a single OSCE case in both an in-person and telemedicine environment provided useful comparisons and helped identify key areas of further training need for the institution's residency program. At the same time, the realities of the larger healthcare environment speak to the need to quickly and proficiently develop telemedicine competency and capacity among our trainees and other physicians.

Three areas of competency development need were identified and explored during the OSCE case - technical proficiency; virtual information gathering, including history, collateral information collection, and physical exam; and interpersonal communication skills, both verbal and nonverbal. These reflect the larger telemedicine training literature, which has identified needs for both technical proficiency and care delivery quality assurance at both trainee and practitioner levels ${ }^{11-}$ 
13. While residents were by-and-large of the "digital native" generation, this did not universally translate into competency with the telemedicine setup or space, or with facility in communicating with patients about telemedicine ${ }^{14}$. Few learners took the opportunity to set the stage for their patient by introducing them to the novel clinical context, discussing the various aspects (including limitations) of the environment, or assessing for patient understanding or concerns. Learners themselves were often unaware of, or did not fully utilize, the tools available to them in the virtual case. At the same time, both the patient and the learners identified challenges around communication and connection; for the SP, the same structured encounter felt subjectively different in a virtual space as compared with an in-person one, and for learners (most of whom had experienced prior in-person OSCEs in their training), the novel context was both instructive and disruptive to their relationship with the patient. This has important implications for patient-provider relationships, including rapport- and trust-building, medication adherence, patient education, and shared decision-making.

There are several limitations to this case study. It was not originally designed as a telemedicine-based encounter; SP and faculty assessments of telemedicine competencies were adapted to the case in a limited time frame and without the same pre-deployment review that generally accompanies OSCE development, including SP and faculty training in use of the evaluation tools. As a result, important telemedicinespecific domains for the case may not have been thoroughly evaluated. Similarly, although resident learners had been informed of the transition to the telemedicine-based platforms and the general expectations around telemedicine care prior to the case, they may not have been fully prepared for the new environment and may have felt uncertain or uncomfortable addressing issues that arose. Unfamiliar setups and workflows may have made it difficult for learners to know what tools were available to aid in their patient evaluations, and technical issues may have been considered the responsibility of the OSCE facilitators or technical staff and therefore not directly addressed.

\section{Recommendations for the Training of Residents During the COVID-19 Period}

Programs interested in building capacity among residents and other trainees to perform telemedicine, particularly in the context of rapid development and deployment during COVID-19, can make a significant impact in their trainees' comfort and preparedness by addressing key issues identified in this case study. At our institution, as a result of this OSCE experience, we instituted a follow-up just-in-time (JIT) teaching session for interested residents, and developed a series of telemedicine best-practice guidelines (see Appendix 3). These guidelines were adapted from both internal institutional best practices for practicing providers as well as recommendations from major medical organizations such as the AMA,
American College of Physicians (ACP), and the American Telemedicine Association (ATA) ${ }^{15-17}$. The guidelines were further refined in collaboration with the participating residents, with plans to disseminate more broadly to the trainee community. As our institution and others across the nation rapidly move to embrace telemedicine as a means to provide safe and comprehensive patient care, we are now better able to equip our trainees with essential skills.

In addition to practical guidelines for those undertaking telemedicine practice, there is urgent need for the rigorous translation and evaluation of "core" trainee competencies into virtual care environments. Until recently, the majority of telemedicine curricula and learner evaluation has focused on technical proficiency or adapted history and exam skills ${ }^{18-20}$. While domains such as professionalism, communication, and other interpersonal skills are often asked of evaluators as part of a general evaluation of trainees, these domains are rarely explicitly adapted to the virtual encounter. Further, there is as yet limited understanding of how to adapt both core competencies and the tools used to evaluate them to technologically mediated contexts such as virtual environments - for example, validated frameworks for building nonverbal communication skills in trainees may need to be reconsidered when they are "virtualized," and the tools used to gauge learners in this area rebuilt and revalidated. In particular, there is recognition of the need for more emphasis on "digital professionalism" for providers and trainees ${ }^{21}$. During this period-as patients look to providers not only for medical information but also for confidence, reassurance, and emotional support-programs must ensure that concepts such as digital professionalism are incorporated into pragmatic telemedicine training. These concepts must also be more fully explored and integrated into general virtual and digital health training in the future. Doing so will help ensure that the future of virtual healthcare includes not only digital savvy but humility and humanity as well.

Acknowledgments: The authors would like to acknowledge and thank the Standardized Patients, trainees, and faculty who contributed to this project, and The New York Simulation Center for the Health Sciences (NYSIM), a Partnership of The City University of New York and NYU Langone Health, who provided material support of staff, space, and equipment for this educational event.

Corresponding Author: Katharine Lawrence, MD MPH; Department of Population Health NYU Grossman School of Medicine, New York, NY, USA (e-mail: katharine.lawrence@nyulangone.org).

Funding Information This project was supported by Agency for Healthcare Research and Quality Grants 11-01865 and T32HSO26120, and Health Resources and Services Administration Grant TOBHP285770100.

\section{Compliance with Ethical Standards:}

Conflict of Interest: The authors declare that they do not have a conflict of interest.

Disclaimer: The content is solely the responsibility of the authors and does not necessarily represent the official views of the Agency for 
Healthcare Research and Quality or the Health Resources and Services Administration.

\section{REFERENCES}

1. Centers for Medicare and Medicaid Services. Medicare Telemedicine Health Care Provider Fact Sheet. https://www.cms.gov/newsroom/factsheets/medicare-telemedicine-health-care-provider-fact-sheet. Accessed $3 / 20 / 2020$.

2. Accreditation Council for Graduate Medical Education. ACGME Response to the Coronavirus (COVID-19). https://acgme.org/Newsroom/ Newsroom-Details/ArticleID/10111/ACGME-Response-to-the-Coronavirus-COVID-19. Access 3/18/2020.

3. Moore, MA, Coffman, M, Jetty, A. Only 15\% of FPs report using telehealth; training and lack of reimbursement are top barriers. Am Fam Physician. 2016 Jan 15;93(2):101.

4. Moore, MA, Jetty, A, Coffman, M. Over half of family medicine residency program directors report use of telehealth services. Telemed $\mathrm{J}$ E Health. 2019 Oct;25(10):933-939.

5. Darkins, A. The growth of telehealth services in the veterans health administration between 1994 and 2014: a study in the diffusion of innovation. Telemed e-Health 2014; 20(9): 761-768.

6. Bell, S, Delbanco, T, Walker, J. OpenNotes: how the power of knowing can change health care. NEJM Catalyst. https://catalyst.nejm.org/doi/ full/10.1056/CAT.17.0372. Accessed 3/18/2020.

7. Webster, $\mathbf{K}$, Tan, A, Unger, $\mathbf{N}$, Lazzara, E. Considerations and Strategies for Assessing: Simulation-Based Training in Interprofessional Education, Comprehensive Healthcare Simulation: InterProfessional Team Training and Simulation, 10.1007/978-3-030-28845-7_9, (121-133), (2020).

8. American Telemedicine Association Resources. https:// www.americantelemed.org/resource/. Accessed 3/18/2020.

9. Miller, E. Telemedicine and doctor-patient communication: an analytical survey of the literature. (2001). Journal of Telemedicine and Telecare, 7(1), 1-17.

10. Miller, E. The technical and interpersonal aspects of telemedicine: effects on doctor-patient communication. (2003). Journal of Telemedicine and Telecare, 9(1), 1-7.
12. Waseh S, Dicker AP. Telemedicine training in undergraduate medical education: mixed-methods review. JMIR Med Educ. 2019;5(1):e12515. Published 2019 Apr 8.

13. Reising, J. Pilot program trains residents in telemedicine. The Hospitalist. https://www.the-hospitalist.org/hospitalist/article/200788/business-medicine/pilot-program-trains-residents-telemedicine. Accessed 3/20/2020.

14. Pathipati AS, Azad TD, Jethwani K. Telemedical education: training digital natives in telemedicine. J Med Internet Res 2016;18(7):e193

15. American Medical Association. AMA quick guide to telemedicine in practice. https://www.ama-assn.org/practice-management/digital/ ama-quick-guide-telemedicine-practice. Access 3/20/2020.

16. American College of Physicians. ACP Telehealth Resources. https:// www.acponline.org/practice-resources/business-resources/health-information-technology/telehealth. Accessed 3/20/2020.

17. American Telemedicine Association. Practice Guidelines and Resources. https://thesource.americantelemed.org/resources/telemedicine-practice-guidelines. Accessed 3/19/2020.

18. Cantone RE, Palmer R, Dodson LG, Biagioli FE. Insomnia telemedicine OSCE (TeleOSCE): a simulated standardized patient video-visit case for clerkship students. MedEdPORTAL. 2019;15:10867.

19. Sartori DJ, Olsen S, Weinshel E, Zabar SR. Preparing trainees for telemedicine: a virtual OSCE pilot. Med Educ. 2019 May;53(5):517-518.

20. Kirkland EB, DuBose-Morris R, Duckett A. Telehealth for the interna medicine resident: a 3-year longitudinal curriculum. J Telemed Telecare. 2019 Dec 31:1357633X19896683.

21. Association of American Medical Colleges. Digital Professionalism. GIR Member Viewpoint, July 2011. https://www.aamc.org/professional-development/affinity-groups/gir/viewpoint-digital-professionalism. Accessed 3/21/2020.

Publisher's Note Springer Nature remains neutral with regard to jurisdictional claims in published maps and institutional affiliations. 